\title{
Interim statement on anti-dementia drugs
}

\section{Implications, concerns and policy proposals}

\begin{abstract}
The availability of anti-dementia drugs inevitably raises great hopes in all those touched by dementia (Kelly et al, 1977) and the Royal College of Psychiatrists welcomes the licensing of these treatments. From this starting point increasing progress is likely to be made in evaluating their effectiveness and usefulness in care. It is important that the best use is made of such treatment, that it is properly monitored and evaluated, and that the many implications for service delivery are adequately addressed. It is essential that the concerns of patients and carers are addressed and that unrealistic hopes are not raised only to be cruelly dashed. The use of these drugs heralds a major change and positive opportunity for the profession.
\end{abstract}

\section{Background issues \\ Effectiveness}

Significant effects of some preparations have been demonstrated but these remain limited. How real will be the impact on care needs in dementia, other than perhaps by delaying them. is unclear. Whether the 'cost' of the illness in an individual case is reduced, either in financial 'burden' or clinical terms remains to be determined.

\section{Side-effects}

Newer generation drugs are reported to have lower rates of side-effects, such as hepatic toxicity and nausea. However, side-effects remain a concern.

\section{Longer-term effects and interactions}

As there has been little experience of their use, adequate monitoring of these aspects will be essential.

\section{Ethics}

Many issues arise and, as yet, have been little addressed. Difficulties with consent and fair use of resources are the most obvious. Whether such treatment 'inappropriately' prolongs life with dementia may be an issue.

\section{Control}

In the light of these concerns, consideration needs to be given as to how their use, initially at least, should be controlled, monitored and funded. Central guidance would be helpful.

\section{Immediate- and medium-term issues}

\section{Who prescribes?}

Advice on prescription should be sought from secondary care specialists by primary care. While clinical experience of these agents is being acquired, initiation of prescribing should be by those with special experience of dementia, such as specialists in Old Age Psychiatry, Geriatric Medicine or Neurology.

If only limited numbers can be treated (as is likely with National Health Service funded treatment) those most likely to benefit should be targeted. Potential difficulties arising from fundholding and the risk of a two tier system of funding treatment should be faced. Accurate assessment, follow-up and careful monitoring to ensure effectiveness, efficacy and safety are required. Monitoring is also required to ensure that sizeable groups of patients have their progress assessed and compared.

Who assesses - who monitors?

For similar reasons this should be done by specialist services. Clinical audit should be engaged in setting standards, monitoring, examining practice and evaluating outcomes. The College proposes to consider the facilitation of such activities. A multi-centre approach would be important. Guidance on the range of investigations for assessment (including psychometry and neuroimaging). monitoring and the development of more sensitive outcome measures will evolve.

As yet there is no evidence that current good practice in assessment (Royal College of Psychiatrists, 1996) should be changed. Early monitoring to ensure tolerance should occur as early as necessary. The optimum frequency of further monitoring has not been agreed. 
Who is to be treated?

Treatment could be appropriate for dementia sufferers of any age who meet defined clinical criteria (e.g. ICD-10 (World Health Organization, 1992)). To date, effects have been noted only for mild to moderate Alzheimer's disease (e.g. MiniMental State Examination score of 10 or more (Folstein et al, 1975)). The difficulty of accurate clinical diagnosis is acknowledged. Significant physical illness may mean treatment should not be given. Sufficient supervisory carer support will be necessary.

The College advocates the development of clinical guidelines to assist with the targeting of treatment and evaluation of effectiveness. Liaison with primary care is essential. The agreement of consensus guidelines, preferably at national, but certainly at regional level, should be an aim.

\section{Who consents?}

Consent in broad terms should be obtained from the patient. Careful thought will be needed where capacity to consent seems in doubt and consideration given to the applicability of consent guidelines associated with mental health legislation and related law.

\section{When should treatment be discontinued?}

No long-term data exist and studies are required. Continued deterioration or increase in rate of deterioration while on treatment suggests lack of clinical effectiveness. Empirical assessment of the impact of withdrawal (perhaps for a brief period) will probably be necessary.

\section{Who provides the service and how?}

Local agreement over which specialists take lead responsibility for assessment, monitoring and evaluation will be essential. Local negotiations over whether maintenance treatment is prescribed by primary care are needed. The implications for service delivery will require local debate and addressing through the contracting process - but national and regional level guidance would be important. Substantial changes in work pattern and work load will occur should it be demonstrated that these agents can play a significant role in the management of Alzheimer's disease.

\section{Funding issues}

Most patients treated will still be living in the community and any benefits in reduced dependency will have consequences for primary care and social services. Sharing of the costs between primary and secondary care seems one area for negotiation. The risk of necessary services being withdrawn from very disabled and needy cases to fund new treatments of limited effectiveness must be guarded against.

\section{References}

Folstein, M. F., Folstein. S. E. \& McHugh, P. A. (1975) Mini-mental state. A practical method for grading the cognitive state of patients for the clinician. Journal of Psychiatric Research, 12, 189-198.

Kelly, C. A., HARVEY, R. I. \& CAYTON, H. (1977) Drug treatments for Alzheimer's disease. British Medical Journal. 314, 693-694.

Royal College OF Psychiatrists (1996) Consensus Statement on the Assessment and Investigation of an Elderly Person with Suspected Cognitive Impairment by a Specialist Old Age Psychiatry Service (Council Report CR49). London: Royal College of Psychiatrists.

WORLD HEALTH ORGANIZATION (1992) The ICD-10 Classification of Mental and Behavioural Disorders: Clinical Descriptions and Diagnostic Guidelines. Geneva: WHO.

WORKING GROUP OF THE COLLEGE'S SECTION FOR THE PSYCHIATRY OF OLD AGE comprising Dr Rob Jones (Chair), Dr Stephen Compton, Dr Cait Goddard, Dr Andrew Fairbairn (DoH Observer) and Dr Liz Gregg

Approved by Council, 28 April 1997. 